\title{
From nature to computing and back
}

\author{
Ke TANG ${ }^{1, \dagger}$, Xin YAO $^{2, \dagger}$ \\ 1 Nature Inspired Computation and Applications Laboratory (NICAL), Department of Computer Science and Technology, \\ University of Science and Technology of China, Hefei 230027, China \\ 2 The Centre of Excellence for Research in Computational Intelligence and Applications (CERCIA), School of Computer Science, \\ University of Birmingham, Birmingham B15 2TT, UK
}

(c) Higher Education Press and Springer-Verlag 2009

Nature has been the source of inspiration for many computational algorithms and promoted the emergence of an interdisciplinary research field called nature inspired computation, which studies computational systems and methodologies that are inspired by and/or borrow ideas from natural systems. In the past decades, scientific research in nature inspired computation has evolved substantially and diverse field of research topics have arisen. On one hand, a variety of novel computational techniques haven been developed via mimicking the behaviors of natural systems, e.g., evolutionary computation, neural computation, swarm intelligence, etc. On the other hand, the developments of these computational techniques result in powerful tools for understanding the nature, and thus benefit the advance of related fields, such as biology, chemistry and material science. This special section aims at bringing together some of the latest results that have been obtained in both directions, i.e., from nature to computing and back.

This special section was initially motivated by two recent workshops concerning nature inspired computation and its applications. The first one, the 2008 UK-China Joint Workshop on "From Nature to Computing and Back", was held on 21-24 February 2008 in Shanghai, China. The second one, the 2008 International Workshop on Nature Inspired Computation and Applications, was held on 27-29 May 2008 in Hefei, China. Participants of the two workshops were invited to submit their original work to the Frontiers of Computer Science in China journal. After two rounds of rigorous reviews by at least two anonymous reviewers, 10 papers were finally accepted, covering a wide range of topics in nature inspired computation, including introduction to promising research directions, analysis and development of existing techniques, and devising novel computational approaches to real-world problems.

It is unsurprising that many real-world problems are associated with multiple incommensurable objectives aroused by different considerations. One common approach to this kind of problems is to integrate the objectives together. Jin et al., in contrast, stated that different objectives should be kept separate, and Pareto analysis serves as a promising way to handle these objectives simultaneously. Through three case studies, they show that Pareto analysis may not only benefit investigating the redundant genetic representation of an evolutionary system, but also help constructing better learning systems such as neural networks.

The paper by Coello Coello is closely related to the first paper in the sense that both papers concern multiobjective problems. The difference is that the former mainly focuses on the nature inspired techniques that have been developed for such problems. In particular, Coello Coello presented a nice review of the evolution of nature inspired techniques for multi-objective optimization, and further discusses the potential areas for further research.

Particle swarm optimization (PSO) is a powerful problem-solving algorithm that was initially developed by simulating the process of birds seeking food. Like many other population-based optimization algorithms (e.g., evolutionary algorithms), diversity among the individuals plays an important role in preventing PSO from being trapped in a local optimum. Shi and Eberhart suggested that the diversity in a population of particle could be

${ }^{\dagger}$ Prof. Ke TANG and Xin YAO are the guest editors of the special section on From nature to computing and back.

E-mail: ketang@ustc.edu.cn,X.Yao@cs.bham.ac.uk 
monitored from different perspectives, including population position diversity, population velocity diversity and cognitive diversity. All these types of diversity together provide us a full picture of the dynamics of PSO. Besides, a number of diversity metrics were also proposed.

Real-world problems are full of constraints. Algorithms that were originally proposed for unconstrained optimization problems might not be effective for constrained optimization problems (COPs). Wang and Cai proposed a variant of PSO, namely hybrid multi-swarm particle swarm optimization (HMPSO), to cope with COPs. The efficacy of HMPSO was evaluated on a large number of benchmark functions.

One major concern about nature inspired computation is to generate novel techniques for important real-world applications. Tsang presented an overview of the application of computational intelligence to stock market forecasting. The paper starts from introducing the general problem background. Then, an approach named EDDIE (Evolutionary Dynamic Data Investment Evaluator), which uses genetic programming to learn functions that identify investment opportunities, is described in detail to demonstrate the potential of computational intelligence for forecasting the stock market.

Search-based software engineering has recently emerged as an important research topic. Search heuristics, such as evolutionary algorithms (EAs), are deemed to be potential tools for automating many software engineering tasks. Both experimental and theoretical analyses are needed to design and evaluate search heuristics before applying this approach to a software engineering problem. The paper by Lehre and Yao presents several new theoretical results regarding the runtime of search heuristics on software engineering problems. First, it is shown that crossover operators might be essential for conformance testing of Finite State Machines, while simple EAs without crossover operator can be ineffective for this problem. Second, different search heuristics, including random search (RS), hill climber (HC) and the alternating variable method (AVM), are compared on the structural software testing problem. It is shown that RS needs the longest expected time to find the optimum, while AVM requires the shortest expected time.

The paper by Quek et al. proposes using evolutionary algorithms to develop a competitive computer player for the one versus one Texas Hold'em poker. The player is evolved with respect to a novel fitness function. Benchmark study against two best heads-up Texas Hold'em A.I. to date verified that evolutionary algorithms are effective in creating competitive strategies in the absence of expert knowledge.

Different from other papers in this special issue, the paper by Wu and Voit concerns constructing computational models for biological systems, rather than borrowing ideas from the biological domain to develop new computational techniques. Modeling frameworks being capable of combining deterministic and stochastic, discrete and continuous, as well as multi-timescale features are of particular interests. Wu and Voit proposed such a framework based on the combination of Biochemical Systems Theory (BST) and Hybrid Functional Petri Nets (HFPN). The proposed framework is described in detail on the example of a toggle switch model.

Evolutionary optimization algorithms have been proved very useful tools in many areas of materials science and chemistry. The paper by Tremayne et al. investigated the application of cultural differential evolution (CDE), a variant of the classical differential evolution (DE) algorithm, to molecular crystallography. The CDE was applied to crystal structure determination from powder diffraction data, and a range of algorithm control parameters were tested so as to fully evaluate the performance of CDE. Empirical results show that CDE gained $40 \%$ improvement in efficiency over the classical DE.

Prior knowledge is always of great help to successfully solving real-world problems, especially when combining with a powerful technique in appropriate ways. Lu et al. provided a case study to show the advantages of integrating prior knowledge with the Min-Max modular network ( $\mathrm{M}^{3}$ network) on a large-scale Japanese patent classification problem. Two kinds of prior knowledge, including patent's publishing date and the hierarchical structure of patent classification system, were taken into account, and the integration of prior knowledge was implemented in the way of splitting training data into subsets that are fed into the $\mathrm{M}^{3}$ network. Experimental studies showed that incorporation of such prior knowledge leads to improved generalization performance in comparison to the $\mathrm{M}^{3}$ network without prior knowledge.

This special section will not be possible without the support from the authors, reviewers and members of the guest editorial board. We would like to take this opportunity to acknowledge great support from the members of the guest editorial board: Yaochu Jin, Yuhui Shi, Kay Chen Tan and Tina Yu. We would also like to express our 
gratitude to the following reviewers: Tianshi Chen, Aniko Ekart, Bin Li, Baoliang Lu, Wenjian Luo, Ponnuthurai Nagaratnam Suganthan, Zhenyu Yang, Maryjane Tremayne, Sihai Zhang. Last but not least, we are very grateful to the organizations that have kindly provided partial financial support to the above-mentioned two workshops, including the National High Performance Computing Center (Hefei) of China, Anhui Province Key Laboratory for Computing and Communication Software Engineering, the Fund for Foreign Scholars in University Research and Teaching Programs (B07033), the National Natural Science Foundation of China (Grant No. 60533020), the CAS-MPG Partner Institute for Computational Biology (PICB), Chinese Academy of Sciences, and the Royal Society (UK) under a grant to Xin Yao in its UK-China Science Network program. 